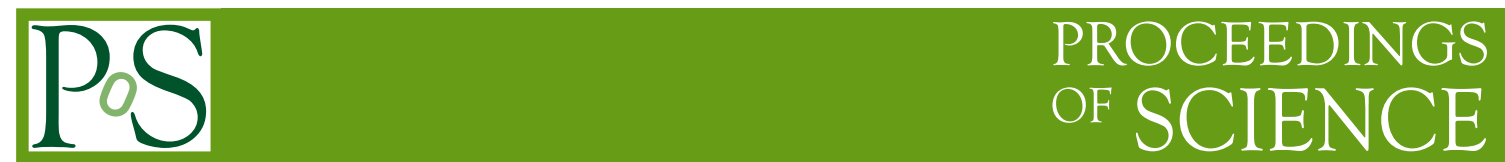

\title{
EWSB scenarios at 2004
}

\author{
Alex Pomarol* \\ IFAE, Universitat Autònoma de Barcelona, \\ 08193 Bellaterra, Barcelona \\ E-mail: pomarol@ifae.es
}

\begin{abstract}
Scenarios of electroweak symmetry breaking (EWSB) are strongly constrained by the electroweak precision data, mostly from LEP, suggesting a little hierarchy between the electroweak scale and the new physics scale. I present two recent scenarios of EWSB arising from extra dimensions that address this little hierarchy and show how well they pass the EW precision tests.
\end{abstract}

28th Johns Hopkins Workshop on Current Problems in Particle Theory

June 5-8, 2004

Johns Hopkins University Homewood campus - Bloomberg Center for Physics and Astronomy, Baltimore, Maryland

${ }^{*}$ Speaker. 


\section{The "holographic" approach in theories with extra compact dimensions}

Starting from the pioneering work of Scherk and Schwarz in 1979 [1], extra dimensions have provided new possibilities to break symmetries. Especially in the recent years theories with more than 4 dimensions have received a lot of attention due to their possible applications to particles physics.

The historical approach to these theories has consisted in decomposing the extra-dimensional fields in infinite series of 4D mass-eigenstates, the Kaluza-Klein (KK) modes, and calculate with them physical quantities. Nevertheless, this KK approach is not always very transparent, lacking sometimes of an intuitive understanding of the results. This is especially true in the case of a warped extra dimension [2]. For this purpose the holographic or boundary procedure is much more useful. It consists in separating the bulk fields from their boundary value and treating them as distinct variables. Three important benefits emerge from this approach:

1. The bulk and the boundary usually respect different symmetries. Therefore it is useful to treat these two sectors separately if we want to keep track of the symmetries of the model. On the other hand, KK modes, being a mixture of boundary and bulk fields, do not have welldefined symmetry transformations and then symmetries in this approach are not manifest.

2. Whenever the bulk fields are weakly coupled to the boundary fields, one can treat the bulk as a small perturbation to the boundary. This occurs in $\mathrm{AdS}_{5}$ spaces. In this case one can perform an expansion in the boundary-bulk couplings (apart from the ordinary expansion in the 5D bulk coupling) that enormously simplify the calculations. An example is the one-loop running of the gauge coupling in $\mathrm{AdS}_{5}$ models, where the obscure KK calculation becomes strikingly simple if one uses the holographic approach [3].

3. The $5 \mathrm{D}$ bulk coupled to the $4 \mathrm{D}$ boundary has certain similarities to a $4 \mathrm{D}$ strongly coupled field theory (SCFT) with a large number of "colors" coupled to some external fields. These similarities are useful to get a simple understanding of the 5D calculations.

Let us elaborate a little bit more on the holographic approach. Consider a five-dimensional theory with metric

$$
d s^{2}=a(z)^{2}\left(\eta_{\mu v} d x^{\mu} d x^{\nu}-d z^{2}\right) \equiv g_{M N} d x^{M} d x^{N},
$$

where the fifth dimension $z$ is compactified on a manifold with boundaries at $z=L_{0}$ and $z=$ $L_{1}\left(L_{0} \leq z \leq L_{1}\right)$. These boundaries will be called ultraviolet (UV) boundary and infrared (IR) boundary respectively. The conformal length of the extra dimension is given by $L=\int_{L_{0}}^{L_{1}} d z=$ $L_{1}-L_{0}$. We are interested in obtaining the partition function $z$ of this theory at the leading order in a semiclassical approximation (tree-level). We proceed in the following way. We first integrate over the bulk fields, $\Phi$, constrained to the UV-boundary value $\Phi\left(x, z=L_{0}\right)=\Phi^{0}(x)$ :

$$
z\left[\Phi^{0}\right]=\int_{\Phi^{0}} d \Phi e^{i S(\Phi)}=e^{i S_{\mathrm{eff}}\left(\Phi^{0}\right)} .
$$

This is done simply by obtaining $\Phi$ from its 5D equation of motion and substituting it back into the action. The boundary condition (b.c.) of $\Phi$ at the IR-boundary must be taken to be consistent with 
the $5 \mathrm{D}$ variational principle. For example, for a scalar field we have ${ }^{1}$

$$
S(\Phi)=\frac{1}{g_{5}^{2}} \int d^{5} x \sqrt{g} \frac{1}{2} g^{M N} \partial_{M} \Phi \partial_{N} \Phi+\cdots
$$

In Eq. (1.3) a coefficient $1 / g_{5}^{2}$ has been factored out in front of the action, so that $g_{5}$ is the $5 \mathrm{D}$ expansion parameter. In order to solve the equations of motion it is useful to work in Euclidean momentum representation along the four non-compact dimensions: $x_{\mu} \rightarrow q_{\mu}$. The general bulk solution in flat space at the leading order in $g_{5}$ (free scalar theory) has the form $\Phi=A(q) \cosh (q z)+$ $B(q) \sinh (q z)$ where $A$ and $B$ are two integrations constants to be fixed by boundary conditions. Demanding $\Phi\left(z=L_{0}=0\right)=\Phi^{0}$ and a Neumann b.c. on the IR-boundary, $\partial_{z} \Phi\left(z=L_{1}=L\right)=0$, we obtain: $\Phi=\Phi^{0}[\cosh (q z)-\tanh (q L) \sinh (q z)]$. Inserting the solution back into the action, we get $^{2}$

$$
S_{\mathrm{eff}}=\frac{1}{g_{5}^{2}} \int_{\mathrm{UV}} \frac{1}{2} \Phi \partial_{z} \Phi=\int \frac{d^{4} p}{(2 \pi)^{4}} \frac{1}{2} \Phi^{0} \Sigma(q) \Phi^{0}, \quad \text { where } \quad \Sigma(q)=-\frac{q}{g_{5}^{2}} \tanh (q L) .
$$

The resulting effective action $S_{\text {eff }}$ is a 4D non-local action of the UV-boundary field $\Phi^{0}$.

As a second step to obtain $Z$, we must integrate over all possible $4 \mathrm{D}$ field configurations $\Phi^{0}$ :

$$
z=\int d \Phi^{0} e^{i\left[S_{\mathrm{eff}}\left(\Phi^{0}\right)+S_{\mathrm{UV}}\left(\Phi^{0}\right)\right]}
$$

where $S_{\mathrm{UV}}$ contains possible local terms for $\Phi^{0}$ on the UV-boundary. If the terms in $S_{\mathrm{UV}}$ dominate over those of $S_{\text {eff }}$, that happens for $L / g_{5}^{2} \ll 1$, the effective theory corresponds to the $4 \mathrm{D}$ action $S_{\mathrm{UV}}$ of the scalar $\Phi^{0}$ with a small correction to its self-energy $\Sigma(q)$ coming from the bulk - Eq. (1.4). In this case $\Phi^{0}$ is approximately a mass-eigenstate.

In $\mathrm{AdS}_{5}$ spaces,

$$
a(z)=\frac{1}{k z},
$$

where $1 / k$ is the AdS curvature radius, one can show that the effect of increasing the length of the extra dimension by moving the UV-boundary $L_{0} \rightarrow L_{0} / \omega$ is equivalent at low-energies [5] to the effect of adding some appropriate UV-boundary terms for the fields in the original theory. For example, for gauge bosons the UV-boundary kinetic term that must be added is proportional to $\ln \omega$ and then can be sizable for large $\omega$.

The 4D boundary action obtained in Eq. (1.2) also allows one to establish, at the qualitative level, the following "holographic correspondence": the functional $z\left[\Phi^{0}\right]$ in Eq. (1.2) is equivalent to the generating functional obtained by integrating out a 4D SCFT in the limit of large number of "colors" $N$ :

$$
z\left[\Phi^{0}\right]=\int d \Phi_{\mathrm{SCFT}} e^{i\left[\operatorname{SSFT}_{\mathrm{SC}}+\Phi^{0} O\right]} .
$$

Here the fields $\Phi^{0}$ correspond to external fields coupled to the strong sector through operators $O$ made of SCFT fields. They act like "sources" for correlators of the CFT operators $O$. This

\footnotetext{
${ }^{1}$ For fermions see Ref. [4]

${ }^{2}$ Alternatively, one can easily show that $\Sigma$ is just the inverse of the 5D scalar propagator along the UV-boundary. For Dirichlet b.c. on the IR-boundary, $\Phi\left(z=L_{1}=L\right)=0$, we obtain $\Sigma=-q \operatorname{coth}(q L) / g_{5}^{2}$.
} 
correspondence implies that at the classical level the 5D bulk is equivalent to a 4D SCFT in the large $-N$ limit. In string theory the holographic correspondence has been conjectured to be an exact duality for certain warped geometries [6]. At the field theoretical level we are considering here, however, this 5D/4D correspondence is simply based on the observation that $n$-point functions defined as

$$
\langle O \cdots O\rangle \equiv \frac{\delta^{n} \ln z}{\delta \Phi^{0} \cdots \delta \Phi^{0}}
$$

can be written, both in the 5D theory and in the large- $N$ SCFT, as sums over infinitely narrow states. For a 4D strongly coupled theory, this decomposition directly follows from the large $-N$ limit [7]. From the 5D point of view, on the other hand, $\langle O \cdots O\rangle$ is computed in terms of 5D propagators, and these can be decomposed as an infinite sum over $4 \mathrm{D}$ propagators of KK modes. Then, the $n$-point function $\langle O \cdots O\rangle$ has a similar decomposition as in large- $N$ SCFT. For example, the two-point function can be written as

$$
\langle O(q) O(-q)\rangle=\sum_{i=1}^{\infty} \frac{F_{i}^{2}}{q^{2}+m_{i}^{2}} .
$$

In the $4 \mathrm{D}$ theory one has $F_{i} \propto \sqrt{N}$, while in the 5D theory $F_{i} \propto 1 / g_{5}$. In general, however, we cannot say much about the field content of the SCFT, nor about the nature of the operators $O$ that couple to the external fields $\Phi^{0}$. In fact, it is not at all guaranteed that a 4D SCFT exists, which leads to the same $z\left[\Phi^{0}\right]$ as that of the 5D theory. Therefore, at the field theoretical level, the holographic correspondence stated above should be rather considered as a holographic interpretation: a qualitative 4D description of a five-dimensional effective field theory. This interpretation, however, is very useful to have a clear and quick qualitative understanding of higher-dimensional theories.

More can be elaborated on the $5 \mathrm{D} / 4 \mathrm{D}$ correspondence when the 5D spacetime is AdS. In the decompactified limit $L_{0} \rightarrow 0, L_{1} \rightarrow \infty$, the boundary action $S_{\text {eff }}$ is invariant under conformal transformations due to the AdS isometries [6]. This implies that, in such a limit, the 4D holographic theory is a conformal field theory (CFT), and the operators $O$ can be organized according to their dimension. The momentum scaling of the correlators $\langle O \cdots O\rangle$ is now determined, and this allows us to derive many properties of the low-energy theory based only on dimensional grounds. In this case holography becomes a very useful tool to understand 5D AdS models.

\section{5D scenarios of electroweak symmetry breaking (EWSB)}

The holographic correspondence allows to obtain 5D theories of EWSB mimicking 4D SCFT such as Technicolor models. Contrary to 4D SCFT, however, we can perform calculations in these 5D theories. The guidelines to built these theories are the following: (1) Take the global symmetries of the 4D SCFT as local symmetries of the 5D bulk, (2) reduce the 5D bulk symmetries on the UVboundary to the SM ones by the use, for example, of Dirichlet b.c., (3) break the EW symmetry by a VEV of a scalar $v$ on the IR-boundary. In the limit $v \gg 1 / L$ the Higgs is very heavy and, after integrating it out, we end up with a theory like Technicolor. Notice that the SM gauge bosons are located at a distance $L$ from the IR-boundary where EWSB occurs, and then the SM fields only notice EWSB at (low) energies $\sim 1 / L$ and not at the value of $v$. In the opposite limit, $v \ll 1 / L$, we obtain a theory with a composite light Higgs. 
In the next section we will propose two 5D scenarios of EWSB. Before doing this, we must understand the constraints on these types of models that arise from electroweak precision experiments. This is crucial to know the viability of the models.

\subsection{Electroweak precision tests: the $\widehat{S}, \widehat{T}, W$ and $Y$ parameters}

Any theory of EWSB must give at low-energies small deviations $(1-0.1 \%)$ from the SM predictions. The theories that we want to study in this article are categorized as universal theories; theories in which new physics effects appear in the self-energies of the SM gauge boson $\Pi_{j}(q)$, $i, j=W_{a}, B$. Assuming that the scale of new physics is larger than the EW scale, one can show, based on symmetry principles and absence of fine-tuning, that the effects of universal theories on the experimental data can be parametrized by 4 form factors [8]:

\begin{tabular}{llcc} 
& Form factors & \multicolumn{2}{c}{ custodial $\mathrm{SU}(2)_{L}$} \\
\hline$\widehat{S}=$ & $g^{2} \Pi_{W_{3} B}^{\prime}(0)$ & + & - \\
$\widehat{T}=$ & $\frac{g^{2}}{M_{W}^{2}}\left[\Pi_{W_{3}}(0)-\Pi_{W^{+}}(0)\right]$ & - & - \\
$W=\frac{g^{2} M_{W}^{2}}{2} \Pi_{W_{3}}^{\prime \prime}(0)$ & + & + \\
$Y=$ & $\frac{g^{2} M_{W}^{2}}{2} \Pi_{B}^{\prime \prime}(0)$ & + & + \\
\hline
\end{tabular}

Above we also show the symmetry that each of these form factors preserve. Experimental data, mostly from LEP1 and LEP2, restricts new physics contributions to $\widehat{S}, \widehat{T}, W$ and $Y$ to be quite small. We find [8]

\begin{tabular}{c|cccc}
$m_{h}$ & $10^{3} \widehat{S}$ & $10^{3} \widehat{T}$ & $10^{3} Y$ & $10^{3} \mathrm{~W}$ \\
\hline 115 & $0.0 \pm 1.3$ & $0.1 \pm 0.9$ & $0.1 \pm 1.2$ & $-0.4 \pm 0.8$ \\
800 & $-0.9 \pm 1.3$ & $2.0 \pm 1.0$ & $0.0 \pm 1.2$ & $-0.2 \pm 0.8$
\end{tabular}

Therefore, we can conclude that in a generic universal model, no matter what the Higgs mass is, $\widehat{S}$, $\widehat{T}, W$ and $Y$ must be small, at the $10^{-3}$ level.

\section{(I) Higgsless theories:}

The first example we want to present is a 5D model of EWSB without a (light) Higgs $[9,10]$. As we already mentioned the UV-boundary lagrangian must correspond to the SM model, while the bulk and IR-boundary must be responsible for EWSB. This latter will mimic a Technicolor sector. In order to avoid a too large contribution to $\widehat{T}$ this sector must preserve a custodial symmetry. The 
symmetry patterns is then assumed to be

$$
\begin{array}{cc}
\text { UV - boundary : } & \mathrm{G}_{\mathrm{SM}} \equiv \mathrm{SU}(2)_{\mathrm{L}} \otimes \mathrm{U}(1)_{\mathrm{Y}} \otimes \mathrm{SU}(3)_{\mathrm{c}} \\
\text { 5D Bulk : } & \mathrm{SU}(2)_{\mathrm{L}} \times \mathrm{SU}(2)_{\mathrm{R}} \otimes \mathrm{U}(1)_{\mathrm{B}-\mathrm{L}} \otimes \mathrm{SU}(3)_{\mathrm{c}} \\
\text { IR - boundary : } & \mathrm{SU}(2)_{\mathrm{L}+\mathrm{R}} \otimes \mathrm{U}(1)_{\mathrm{B}-\mathrm{L}} \otimes \mathrm{SU}(3)_{\mathrm{c}}
\end{array}
$$

The SM fermions will arise as the massless modes of 5D fermion fields. By an appropiate choice of the 5D fermion masses, one can show that these massless states can be localized towards the UV-boundary. Therefore the UV-boundary fields correspond to a good aproximation to the SM fields. By integrating out the bulk and IR-boundary we can write the effective theory as a function of the UV-boundary fields. These are the SM gauge fields $W_{a}$ and $B$. We obtain $\mathcal{L}=\mathcal{L}_{\mathrm{SM}}+\mathcal{L}_{\mathrm{eff}}$ where for the transverse gauge bosons at the quadratic level,

$$
\mathcal{L}_{\mathrm{eff}}=-\frac{1}{2} W_{\mu}^{a} \Delta \Pi_{W W}(q) W^{a \mu}-W_{\mu}^{3} \Delta \Pi_{W B}(q) B^{\mu}-\frac{1}{2} B_{\mu} \Delta \Pi_{B B}(q) B^{\mu} .
$$

For flat space the vacuum polarizations $\Delta \Pi_{V}$ are calculated in Ref. [10]. Since the IR-boundary breaks the EW symmetry, the $W$ and $Z$ get a mass:

$$
M_{W}^{2}=-\Delta \Pi_{W W}(0)=2 g^{2} \frac{M}{L}, \quad M=\frac{M_{L} M_{R}}{M_{L}+M_{R}},
$$

where the last relation applies only in 5D flat space and $M_{L, R}=1 / g_{5 L, R}^{2}$ is the inverse 5D gauge coupling squared. The problem of this model is, however, that $M_{W}$ is too close to $1 / L$ and therefore one expects large contributions to the $\widehat{S}, \widehat{T}, W$ and $Y$ parameters. The largest contribution appears in $\widehat{S}$. In flat space one finds

$$
\widehat{S}=g^{2} \frac{4}{3} M L\left[1+\frac{3}{4} \frac{Z_{W}}{\left(M_{L}+M_{R}\right) L}\right],
$$

where $Z_{W}$ is the coefficient of the kinetic term of the $\mathrm{SU}(2)_{\mathrm{L}+\mathrm{R}}$ gauge bosons on the IR-boundary. Notice that $\widehat{S}$ grows with the inverse 5D coupling. More precisely parametrizing the 5D loop expansion parameter as ${ }^{3} \ell_{5}=/\left(48 \pi^{3} M R\right)$ we have

$$
\widehat{S}=\frac{g^{2}}{36 \pi^{2}} \frac{1}{\ell_{5}} .
$$

For $\ell_{5} \ll 1$ (necessary to have a reliable 5D gauge coupling expansion), one has $\widehat{S} \gg 10^{-3}$, indicating that (marginal) agreement with the data can only be obtained in the region where $\widehat{S}$ is not calculable. In this respect a Higgsless theory in 5D does not fare better than a generic strongly coupled and incalculable 4D one. It can be proven that this is true for any 5D metric. Even if the breaking of the electroweak symmetry is broken by a scalar in the bulk instead of by the IRboundary one always obtains a large contribution to $\widehat{S}$.

We must also say that contributions to $\widehat{T}$, arising at the one-loop level [11, 12], are also sizable in these types of models.

\footnotetext{
${ }^{3}$ By applying naive dimensional analysis the scale at which the $5 \mathrm{D}$ theory becomes strongly coupled is $48 \pi^{3} M$. When $\ell_{5} \sim 1$ the theory is strongly coupled already at the energy of the lightest KK mode, so that the 5D description is never valid, and predictivity is totally lost.
} 


\section{(II) Composite Higgs:}

The presence of a light Higgs seems to be a crucial ingredient if we want to avoid large contributions to $\widehat{S}$ and $\widehat{T}$. In this case the Higgs VEV can be tuned to be smaller than $1 / L$ to the desirable value [11]. This fine tuning, however, can be avoided if the Higgs sector is protected by an approximate symmetry. An interesting possibility is to have a Higgs arising as a pseudo-Goldstone boson (PGB). In this case, the Higgs mass is protected by an approximate global symmetry, similarly as pions in QCD. ${ }^{4}$ Furthermore, its potential is completely determined by IR-physics (is induced by loop of SM gauge boson and loop of tops) and the EWSB can be predicted.

Here we want present a 5D model in which the Higgs appears as a PGB, or, equivalently, as the fifth component of a $5 \mathrm{D}$ gauge boson $\left(A_{5}\right)[14,12]$. The symmetry pattern of the model is given by [12]

$$
\begin{array}{cc}
\text { UV - boundary : } & \mathrm{SU}(2)_{\mathrm{L}} \otimes \mathrm{U}(1)_{\mathrm{Y}} \otimes \mathrm{SU}(3)_{\mathrm{c}} \\
\text { 5D Bulk : } & \mathrm{SO}(5) \otimes \mathrm{U}(1)_{\mathrm{B}-\mathrm{L}} \otimes \mathrm{SU}(3)_{\mathrm{c}} \\
\text { IR - boundary : } & \mathrm{SO}(4) \otimes \mathrm{U}(1)_{\mathrm{B}-\mathrm{L}} \otimes \mathrm{SU}(3)_{\mathrm{c}}
\end{array}
$$

This is the minimal scenario that accomplishes three things: it delivers a PGB being a 2 of SU(2), the Higgs, it has a custodial SU(2) $)_{L+R}$ symmetry after EWSB (up to UV-boundary terms), and it contains the SM gauge group. The presence of a massless scalar at tree-level can be explicitly seen by, for example, working in the unitary gauge. In this gauge $A_{5}$ is non-vanishing only in its $\mathrm{SO}(5) / \mathrm{SO}(4)$ components, which are however constrained to have a fixed profile along the fifth dimension: $A_{5}(x, z)=\zeta(z) h(x), \zeta(z)=z \sqrt{2 /\left(L_{1}^{2}-L_{0}^{2}\right)}$ for $\operatorname{AdS}_{5}$ and $\zeta(z)=$ const for flat space. Thus, physical fluctuations of $A_{5}$ correspond to a $4 \mathrm{D}$ scalar field $h(x)$ transforming as a 4 of $\mathrm{SO}(4)$, the Higgs. From the point of view of the 5D theory, a potential for $A_{5}$ is forbidden at tree-level by gauge invariance, but it is generated radiatively due to the presence of the UV-boundary.

The SM fermions are embedded into 5D Dirac spinors $\xi_{i}$ which live in the bulk and belong to the $\mathbf{4}_{1 / 3}$ representation of $\mathrm{SO}(5) \times \mathrm{U}(1)_{B-L}$. By an appropriate determination of the bulk and boundary masses we can obtain a realistic theory of fermion masses. In AdS small fermion masses can be naturally obtained since the Higgs $\left(A_{5}\right)$ is localized towards the IR-boundary. Therefore small Yukawas can be obtained for the 1st and 2nd family by localizing the zero-mode fermions towards the UV-boundary and then having a small overlapping with the Higgs [15]. Interestingly, flavor transitions induced by the KK are small and there is not conflict with the experimental data. This can be easily understood in the holographic approach. The 1st and 2nd family of SM fermions are approximately UV-boundary fields with a very small coupling to the bulk.

By integrating the bulk, we can obtain the effective theory on the UV-boundary. We can write this theory in the background of a constant Higgs $h$. For the transverse part of the gauge bosons the effective lagrangian, at the quadratic level, can be written as

$$
\mathcal{L}_{\mathrm{eff}}=\frac{1}{2}\left[\Pi_{a}(q) A_{\mu}^{a} A^{a \mu}+\Pi_{\hat{a}}(q) A_{\mu}^{\hat{a}} A^{\hat{a} \mu}\right] .
$$

The indexes $a$ and $\hat{a}$ run respectively over the $\mathrm{SO}(4)$ generators (unbroken on the IR-boundary) and

\footnotetext{
${ }^{4}$ Global supersymmetry can be also an example for such symmetry [13].
} 
the $\mathrm{SO}(5) / \mathrm{SO}(4)$ generators (broken on the IR-boundary), and

$$
\Pi_{a, \hat{a}}(q)=-\frac{1}{g_{5}^{2} k} \frac{p}{L_{0}} \frac{Y_{0}\left(q L_{0}\right) \tilde{J}_{0,1}\left(q L_{1}\right)-\tilde{Y}_{0,1}\left(q L_{1}\right) J_{0}\left(q L_{0}\right)}{Y_{1}\left(q L_{0}\right) \tilde{J}_{0,1}\left(q L_{1}\right)-\tilde{Y}_{0,1}\left(q L_{1}\right) J_{1}\left(q L_{0}\right)}
$$

with $\tilde{J}_{1}\left(q L_{1}\right)=J_{1}\left(q L_{1}\right), \tilde{J}_{0}\left(q L_{1}\right)=J_{0}\left(q L_{1}\right)-\left(g_{5}^{2} k / g_{\mathrm{IR}}^{2}\right) p L_{1} J_{1}\left(q L_{1}\right)$ and similarly for $\tilde{Y}_{0,1}$. Here $g_{5}^{2}$ denotes the $\mathrm{SO}(5)$ bulk gauge coupling, and $1 / g_{\mathrm{IR}}^{2}$ is the coefficient of the $\mathrm{SO}(4)$ boundary kinetic term on the IR-boundary. Analogous formulas apply for the $\mathrm{U}(1)_{B-L}$ gauge boson, that has been omitted in Eq. (2.5) for simplicity. We have not written down possible boundary kinetic terms on the UV-boundary, though they can be included in a straightforward way.

From Eq. (2.5) we can get the contribution to $\widehat{S}$ :

$$
\widehat{S}=\frac{g^{2}}{2}\left[\Pi_{\hat{a}}^{\prime}(0)-\Pi_{a}^{\prime}(0)\right] \varepsilon^{2}=\frac{3 g^{2}}{8} \frac{1}{g_{5}^{2} k} \varepsilon^{2}\left[1+\frac{4}{3} z_{\mathrm{IR}}\right],
$$

where $\varepsilon=246 \mathrm{GeV} / f_{\pi}$ with $f_{\pi}^{2}=4 /\left(g_{5}^{2} k L_{1}^{2}\right)$ and $z_{\mathrm{IR}}=g_{5}^{2} k / g_{\mathrm{IR}}^{2}$. In order to satisfy $\widehat{S} \lesssim 2.5 \cdot 10^{-3}$ (the $99 \%$ CL bound), we must require

$$
\varepsilon \lesssim 0.5 \sqrt{\left(\frac{10}{N}\right) \frac{1}{1+4 / 3 z_{\mathrm{IR}}}},
$$

where $N=16 \pi^{2} / g_{5}^{2} k$. We see that in order to remain in the weak coupling regime, $1 / N \ll 1$, we need $\varepsilon<1$. In particular, taking $N=10$ and $z_{\mathrm{IR}}=0$ (1) requires $\varepsilon \sim 0.5(0.3)$. To determine whether EWSB is triggered and an $\varepsilon<1$ is generated in our model, one has to compute and minimize the Higgs potential.

\section{Higgs potential and vacuum misalignment}

At the one-loop level a non-zero Higgs potential is generated. The most important contributions arise from the $\mathrm{SM} \mathrm{SU}(2)_{L}$ gauge boson and from the top. One can show that, to a good aproximation, the Higgs pontetial is given by

$$
V(h) \simeq \alpha \cos \frac{h}{f_{\pi}}-\beta \sin ^{2} \frac{h}{f_{\pi}},
$$

where $\alpha$ and $\beta$ are constants. This potential has a minimum at $\cos h / f=-\alpha /(2 \beta)$, i.e.

$$
\varepsilon=\sqrt{1-\left(\frac{\alpha}{2 \beta}\right)^{2}}
$$

Thus, for suitable values of $\alpha$ and $\beta$ the EWSB can occur dynamically. Gauge fields only contribute to the $\beta$ with an overall positive coefficient and tend to align the vacuum in the $\mathrm{SU}(2)_{L}$ preserving direction. A misalignment of the vacuum can only come from top loops, and only if the coefficients $\alpha$ and $\beta$ are comparable in size. The physical Higgs mass is given by

$$
m_{\mathrm{Higgs}}^{2} \simeq \frac{2 \beta \varepsilon^{2}}{f_{\pi}^{2}} \sim \frac{N_{c}}{N}(246 \mathrm{GeV})^{2}
$$



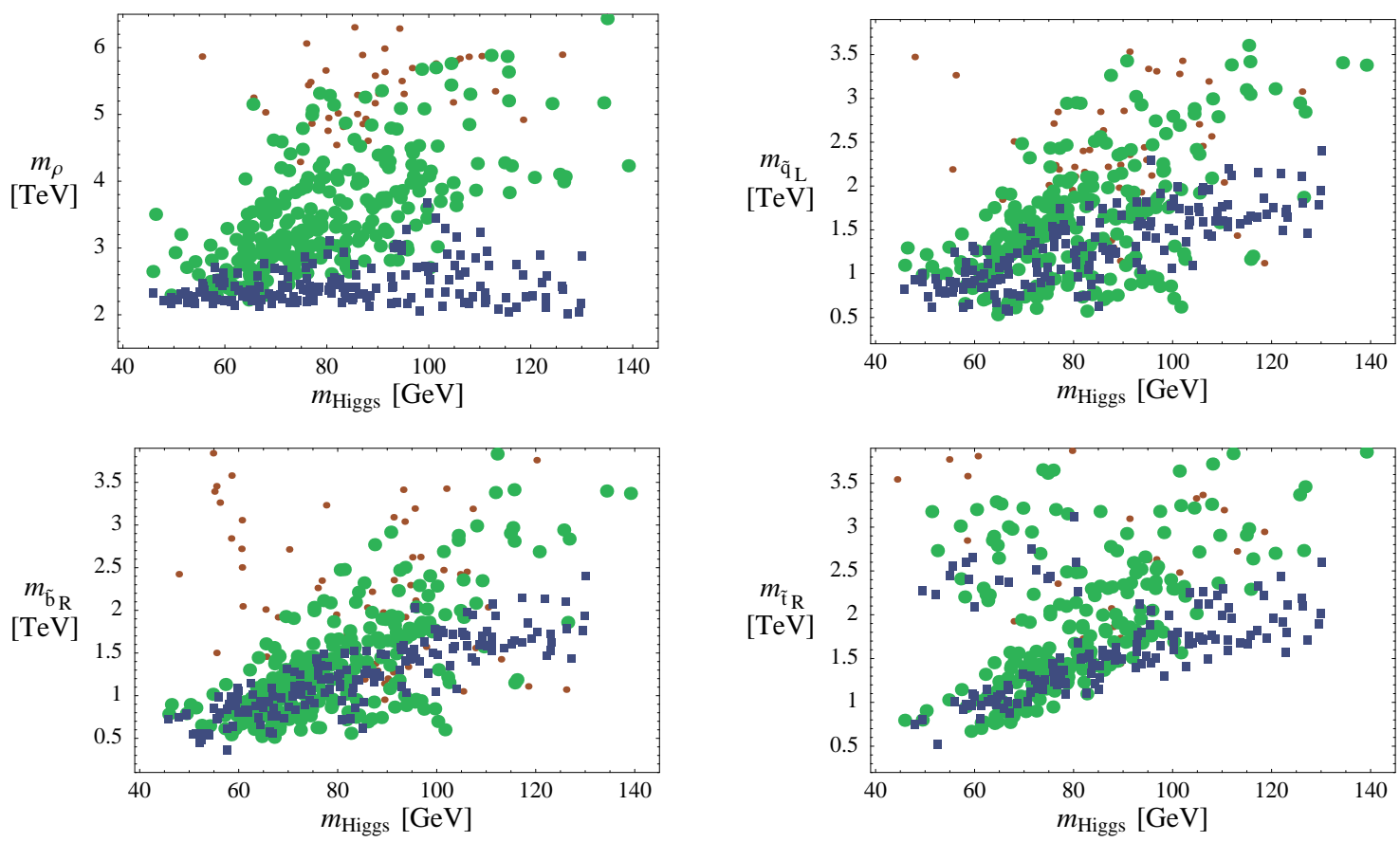

Figure 1: Masses of the first vectorial and fermionic resonances $\rho, \tilde{q}_{L}, \tilde{b}_{R}, \tilde{t}_{R}$ obtained by scanning over the input parameters in the minimal model. Blue squares correspond to $\varepsilon>0.4$, green fat dots to $0.2<\varepsilon<0.4$, small red dots to $\varepsilon<0.2$.

We see that for moderate values of $N$ the Higgs mass can be above the experimental bound $m_{\text {Higgs }}>$ $114 \mathrm{GeV}$. Since in order to satisfy the experimental constraints coming from $\widetilde{S}$ we need $\varepsilon<1$, then, from Eq. (2.10), we see that the relation $\alpha \simeq 2 \beta$ must be fulfilled at the $10 \%$ level for $\varepsilon \lesssim 0.4{ }^{5}$ This is possible by an adjustment of the 5D parameters: the 5D gauge coupling and $5 \mathrm{D}$ fermion masses.

Fig. 1 shows points in the parameter space of the model that pass all EWPT. The figures give the mass of the resonances as a function of the Higgs mass. The fermionic KK states $\tilde{q}_{\mathrm{L}}, \tilde{t}_{R}, \tilde{b}_{R}$ tend to be lighter than the gauge resonances. For values of $m_{\text {Higgs }} \sim 115 \mathrm{GeV}$ and $\varepsilon \sim 0.4$, all fermionic resonances lie around $1.5-2 \mathrm{TeV}$, while $m_{\mathrm{p}} \sim 2-3 \mathrm{TeV}$.

\section{Acknowledgments}

It is a pleasure to thank my collaborators that contributed to the work presented here: K. Agashe, R. Barbieri, R. Contino, T. Gherghetta, Y. Nomura, R. Rattazzi and A. Strumia. I would also like to thank the organizers for their kind invitation. This work was supported in part by the MCyT and FEDER Research Project FPA2002-00748 and DURSI Research Project 2001-SGR-00188.

\section{References}

[1] J. Scherk and J. H. Schwarz, Phys. Lett. B 82, 60 (1979).

\footnotetext{
${ }^{5}$ This is also enough to satisfy the constraints from $T$ and $Z \rightarrow b \bar{b}$.
} 
[2] L. Randall and R. Sundrum, Phys. Rev. Lett. 83, 3370 (1999).

[3] N. Arkani-Hamed, M. Porrati and L. Randall, JHEP 0108, 017 (2001).

[4] R. Contino and A. Pomarol, JHEP 0411 (2004) 058.

[5] A. Lewandowski, M. J. May and R. Sundrum, Phys. Rev. D 67, 024036 (2003).

[6] J. M. Maldacena, Adv. Theor. Math. Phys. 2, 231 (1998) [Int. J. Theor. Phys. 38, 1113 (1999)]; S. S. Gubser, I. R. Klebanov and A. M. Polyakov, Phys. Lett. B 428, 105 (1998); E. Witten, Adv. Theor. Math. Phys. 2, 253 (1998).

[7] G. 't Hooft, Nucl. Phys. B 72, 461 (1974); B 75, 461 (1974); E. Witten, Nucl. Phys. B 160, 57 (1979).

[8] R. Barbieri, A. Pomarol, R. Rattazzi and A. Strumia, Nucl. Phys. B 703 (2004) 127.

[9] C. Csaki, C. Grojean, L. Pilo and J. Terning, Phys. Rev. Lett. 92 (2004) 101802.

[10] R. Barbieri, A. Pomarol and R. Rattazzi, Phys. Lett. B 591, 141 (2004).

[11] K. Agashe, A. Delgado, M. J. May and R. Sundrum, JHEP 0308, 050 (2003).

[12] K. Agashe, R. Contino and A. Pomarol, arXiv:hep-ph/0412089.

[13] T. Gherghetta and A. Pomarol, Phys. Rev. D 67, 085018 (2003).

[14] R. Contino, Y. Nomura and A. Pomarol, Nucl. Phys. B 671, 148 (2003).

[15] T. Gherghetta and A. Pomarol, Nucl. Phys. B 586, 141 (2000). 